\title{
Refining deep convolutional features for improving fine-grained image recognition
}

Weixia Zhang ${ }^{1}$, Jia Yan ${ }^{1}$, Wenxuan Shi ${ }^{2}$, Tianpeng Feng ${ }^{1}$ and Dexiang Deng ${ }^{{ }^{*}}$

\begin{abstract}
Fine-grained image recognition, a computer vision task filled with challenges due to its imperceptible inter-class variance and large intra-class variance, has been drawing increasing attention. While manual annotation can be utilized to effectively enhance performance in this task, it is extremely time-consuming and expensive. Recently, Convolutional Neural Networks (CNN) achieved state-of-the-art performance in image classification. We propose a fine-grained image recognition framework by exploiting CNN as the raw feature extractor along with several effective methods including a feature encoding method, a feature weighting method, and a strategy to better incorporate information from multiscale images to further improve recognition ability. Besides, we investigate two dimension reduction methods and successfully merge them to our framework to compact the final image representation. Based on the discriminative and compact framework, we achieved the state-of-the-art performance in terms of classification accuracy on several finegrained image recognition benchmarks based on weekly supervision.
\end{abstract}

Keywords: Fine-grained image recognition, Convolutional Neural Networks (CNN), Bag-of-visual-words, Feature weighting, Dimension reduction

\section{Introduction}

As a fashionable topic in computer vision, fine-grained image recognition has been attracting increasingly attention from both academia and industry in the past few years. Taking identifying a bird as an example, it not only aims at pointing out whether the input image presenting a bird or something else ('cat,', 'dog,' 'plant,' 'car'etc.), but also the specifies of the bird (a 'albatross' or a 'cuckoo', or even more exact, a 'black footed albatross'), which can only be discriminated by minute difference of physiological feature. Besides, diverse postures, circumstances, viewpoints, positions, etc. may usually cause non-negligible interference for recognition, which further increase the difficulty. Therefore, compared with general object recognition, this task is rather challenging.

In view of the above annoying problems, part annotations, object bounding box or part annotations are often used to eliminate background noise and to highlight the discriminative part [1-6], such as the whole body, head, or torso of a bird. However, these manual labeling works are always extremely time-consuming, expensive, and

\footnotetext{
* Correspondence: wythia1989@gmail.com

'School of Electronic Information, Wuhan University, Wuhan, China

Full list of author information is available at the end of the article
}

not completely accurate due to artificial error or diverse subjective cognition of the exact position information.

Without such expensive manual label information, powerful image representation will be the key factor for fine-grained visual recognition. Convolutional Neural Networks (CNN)-based methods achieve state-of-the-art. FV$\mathrm{CNN}$ [7] is initially proposed for texture recognition and has been shown to be also suitable for fine-grained visual recognition. One most appealing advantage of FV-CNN may be that it can incorporate multi-scale image information seamlessly. Bilinear $\mathrm{CNN}$ model (B-CNN) [8] extract features from non-linearity activations of convolutional layers from two $\mathrm{CNN}$, achieving remarkable performance in fine-grained visual recognition without any boundingbox or part annotation. We propose our fine-grained image recognition framework based on FV-CNN and gear it with a novel strategy of utilizing multi-scale information. B-CNN, however, will be treated as an end-to-end model fine-tuning method in our framework. Besides, we investigate two dimension reduction methods: Tensor Sketch approximation [9] and Mutual Information dimension selection [10] to compact FV-CNN which is rather highdimensional and cannot be generalized to large-scale task. 
Apart from powerful image representation, picking useful features in an unsupervised manner is another path to enhance performance. By an intuitive and heuristic consideration, hand-crafted saliency detection technologies [11] might be a straightforward choice. However, most saliency detection are implemented on pixel level, which might detect salient region in human's perspective, which is not necessary for a fine-grained image recognition system. In a CNN's pipeline, numerous feature maps, which can be spatially mapped back to the original image, will be generated spontaneously. Because the feature maps' values were obtained by an iteratively optimization process which aim at achieving better recognition performance, they can be utilized to locate and extract discriminative descriptors of an image. In this line of thought, several works have already achieved good results [12-14]. In this paper, we propose a nonparametric feature weighting method based on refining convolutional descriptors to boost performance.

Overview of our proposed feature extraction framework is illustrated in Fig. 1.

The remainder of this paper is organized as follows: Section 2 describes related works. Our proposed strategy of utilizing multi-scale information is discussed in Section 3. In section 4, we describe our feature processing in detail. Section 5 describes compact FV-CNN. Experiment results and analysis are given in Sections 6, and Section 7 concludes the paper.

\section{Related works}

\subsection{FV-CNN}

Bag-of-visual-words (BOW) model and its variants such as Fisher Vectors $[15,16]$ and VLAD $[17,18]$, and deep learning led by Convolutional Neural Networks (CNNs) $[19,20]$ are two mainstreaming architecture for image classification. Pipelines of classical BOW and its variants are composed of four steps:

- Firstly, extracting local descriptors, e.g., SIFT [21], HOG [22], LBP [23], etc.

- Secondly, clustering the local descriptors by Kmeans or Gaussian mixture model (GMM) and forms a visual dictionary.

- After that, repeating the first step for every test image and encoding them to a single feature vector based on histogram of occurrences of each terms in visual dictionary or storing the statistics of the difference between centers (K-means) or modes (GMM) and each descriptor.

- Finally, feeding the encoded feature vectors to a classifier such as linear SVM and getting the classification result.

FV-CNN framework extract descriptors from the last convolutional layer of a $\mathrm{CNN}$ to replace hand-crafted features, and the rest steps are similar to classical BOW model.

Although FV-CNN can incorporate multi-scales information, which has been shown to be an effective strategy to boost recognition performance, of an image in a seamlessly manner, its parameters, i.e., parameters of its GMM cannot be tuned like traditional CNN, which limit its performance.

\subsection{Bilinear CNN (B-CNN)}

Similar to FV-CNN, Bilinear CNN (B-CNN) also employs convolutional features from $\mathrm{CNN}$. In $\mathrm{B}-\mathrm{CNN}$, the

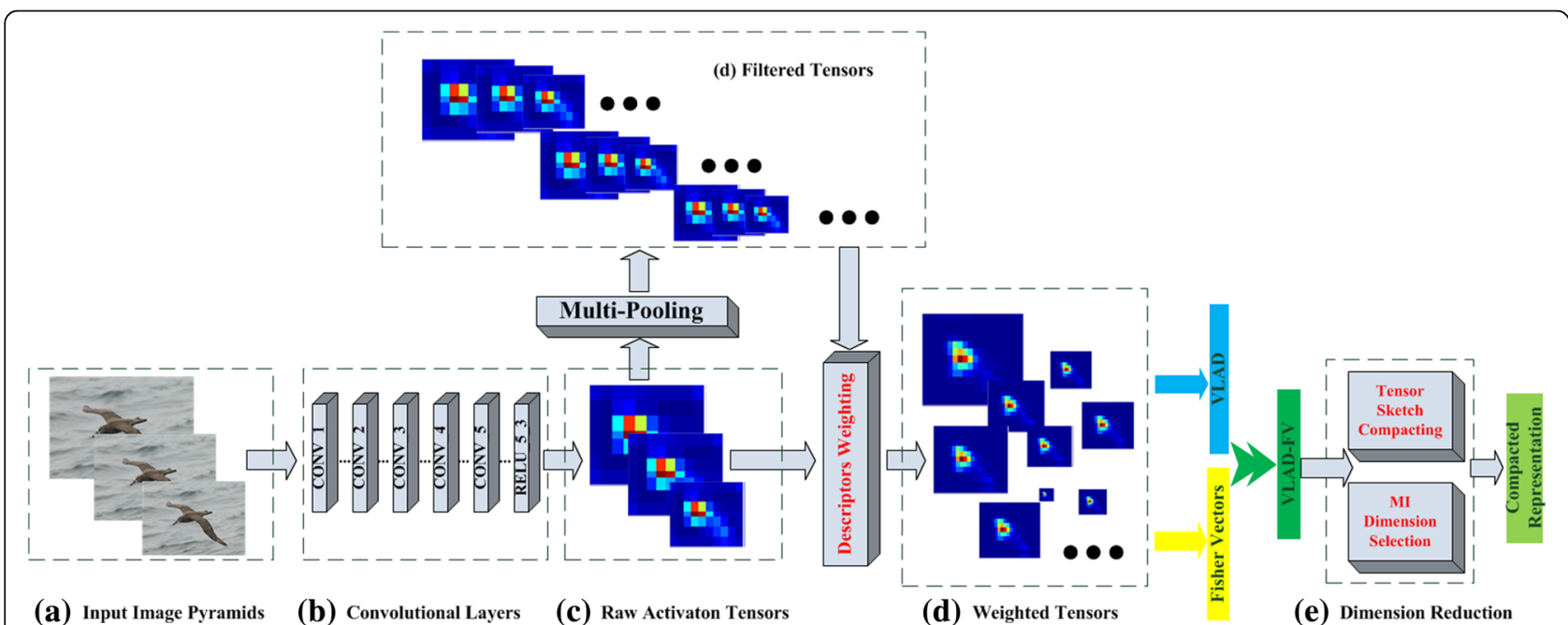

(a) Input Image Pyramids

(b) Convolutional Layers

(c) Raw Activaton Tensors

(d) Weighted Tensors

(e) Dimension Reduction

Fig. 1 An overview of our proposed feature extraction framework. VGG-16 [25] is used as the basic feature extractor. After rectified convolutional descriptors are extracted, they will be refined by a series of operations including multi-pooling, descriptors weighting, concatenation of two representations, and optional dimension reduction module. The panels in the figure refer to different modules in the proposed framework 
feature outputs are combined at each location of feature maps from rectified convolutional layer using the matrix outer product. Unlike FV-CNN, B-CNN can be trained by end-to-end learning, therefore, it also provides an alternative training method to traditional fully connected training (FC-CNN). Lin et al. employ B-CNN [8] to achieve remarkable performance in several fine-grained visual recognition benchmark datasets.

Despite that B-CNN is powerful, its final feature representation requires tremendous storage due to the extremely high dimension (e.g., 262,144 dimensional per image on VGG-16). Gao et al. [24] proposed two compact $\mathrm{B}-\mathrm{CNN}$ methods with the same discriminative power as the full bilinear representation with much less dimensions.

\subsection{Feature weighting}

Unsupervised discriminative region detection is crucial for fine-grained image recognition without object boundingbox and part annotations. Recently, Wei et al. proposed an architecture termed 'Selective Convolutional Descriptor Aggregation (SCDA)' [13] to select useful convolutional descriptor by feature maps from multiple layers in a $\mathrm{CNN}$, which achieved good performance in both finegrained retrieval and fine-grained recognition. Zhang et al. [14] proposed a spatially weighted Fisher Vector (SWFV) for improving the performance of $\mathrm{FV}-\mathrm{CNN}$ in finegrained visual recognition task by spatially weighting Fisher Vectors.

\section{Multi-scale feature}

Inheriting from classical bag-of-visual-words model, FVCNN pools multi-scale information from input image pyramids, i.e., different rescaled versions of a same original image, as shown in the flowing from part (a) to part (c) in Fig. 1. Although this classical technique is effective for improving recognition capability, it meets some limitations. In order to extract features from a $P$-level image pyramids, i.e., image pyramids consist of images with $P$ different resolutions re-scaling from one original image, each of them should be fed into the CNN model and be processed by some preliminary operation such as convolution and pooling layer by layer, as shown in part (b) in Fig. 1, which brings large computation burden as $P$ increases especially in a very deep model such as VGG-16 [25]. Besides, the way of generating multi-scale image features is simply based on resizing the input image by interpolation-based methods such as bilinear interpolation or bicubic interpolation; this is somewhat monotonous in terms of diversity of feature.

Facing these limitations, we propose to generate multiscale information by pooling feature tensors of the last convolutional layer with relu activation in a CNN using different pooling window sizes, which is shown in the flowing of part (c) to part (d) in Fig. 1 and more detailed in Fig. 2. Compared with traditional image resizing method, which inputs a resized image to the $\mathrm{CNN}$, and let it get through all layers in it, this one only get through one layer, i.e., a pooling layer, which needs much less computation, furthermore, the pooling operation is substantially different from interpolation-based image resizing and thus it can enrich the multi-scale information by jointly utilized with the former.

This proposed method can be explained in receptive fields. When a CNN is applied on an image, assume the size of the activation feature maps of last convolutional layer is $H \times W \times D$, where $H$ and $W$ are the height and width of the activation feature maps, respectively, and the $D$ denotes number of feature maps, i.e., the number of channels. This activation structure can not only be seen as $D$ feature maps, but also $H \times W$ spatially distributed $D$ dimensional descriptors. Each descriptor corresponds to a receptive field in the original image. Receptive fields with different sizes covers different content in original image, hence, this operation is in accordance with traditional multi-scale strategy by using image pyramids, both of which will generate more comprehensive information compared with single-scale image or single activation feature map. The multi-pooling and image pyramids can also be jointly utilized in a straightforward way, i.e., applying multi-pooling to the raw activation feature maps of each image from the input image pyramid. For choice of pooling method, we use max-pooling here.

\section{Feature processing}

\subsection{Feature encoding}

Fisher Vectors $[15,16]$ and VLAD $[17,18]$ are two commonly used features encoding in bag-of-visual-words framework. Given a trained Gaussian mixture model

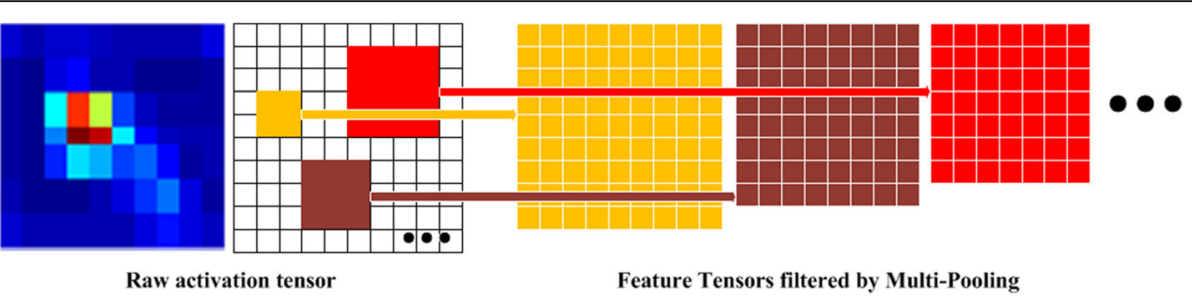

Fig. 2 Illustration of multi-pooling by different pooling window sizes 
(GMM) with parameter $\Theta=\left(\mu_{k}, \Sigma_{k}, \pi_{k} \in \mathbb{R}^{D} ; k=1\right.$, ..., K) and K-means dictionary with centers $c_{1}, \ldots, c_{k} \in \mathbb{R}^{D}$, let $I=\left(x_{1}, \ldots, x_{N}\right)$ be a set of $D$-dimensional feature descriptors extracted from an image, Fisher Vectors pools these descriptors by $\Phi(I)=\left[u_{1}, \ldots, u_{K}, v_{1}, \ldots, v_{K}\right]$, where:

$$
\begin{aligned}
& u_{j k}=\frac{1}{N \sqrt{\pi_{k}}} \sum_{i=1}^{N} q_{\mathrm{ik}} \frac{x_{\mathrm{ji}}-\mu_{\mathrm{jk}}}{\sigma_{\mathrm{jk}}}, \\
& v_{\mathrm{jk}}=\frac{1}{N \sqrt{2 \pi_{k}} \sum_{i=1}{ }^{N} q_{\mathrm{ik}}\left[\left(\frac{x_{\mathrm{ji}}-\mu_{\mathrm{jk}}}{\sigma_{\mathrm{jk}}}\right)^{2}-1\right]} \\
& q_{\mathrm{ik}}=\frac{\exp \left[-\frac{1}{2}\left(x_{i}-\mu_{k}\right)^{T} \Sigma_{k}{ }^{-1}\left(x_{i}-\mu_{k}\right)\right]}{\sum_{\mathrm{t}=1}{ }^{K} \exp \left[-\frac{1}{2}\left(x_{i}-\mu_{t}\right)^{T} \Sigma_{k}{ }^{-1}\left(x_{i}-\mu_{t}\right)\right]} .
\end{aligned}
$$

$o m \sigma_{\mathrm{jk}}$ is just the diagonal element of diagonal matrix $\Sigma_{k}$, and index $j$ span all dimensions. $u_{\mathrm{jk}}$ and $v_{\mathrm{jk}}$ are accumulated sum of the first and second order statistics of descriptors $x_{\mathrm{ji}}$ respectively. Different from Fisher Vectors, VLAD only accumulate the first order statistics with K-means dictionary:

$$
v_{\mathrm{jk}}=\sum_{i=1}^{\mathrm{N}} q_{\mathrm{ik}}\left(x_{\mathrm{ji}}-c_{\mathrm{jk}}\right)
$$

Where $q_{\mathrm{ik}}$ here is usually obtained by nearest neighbor assignment methods such as approximate nearest neighbors (ANN).

Observing (1) and (2), it is clear that Fisher Vectors and VLAD are formulated differently and resort to different visual dictionary, i.e., GMM and K-means, respectively. Instead of researching which encoding method is better, we propose to simply concatenate them to form a new vector which we term as VLAD-FV and can be formulated as:

$$
\varphi(I)=\left[v_{1}, \ldots, v_{K}, u_{1}, \ldots, u_{K}, v_{1}, \ldots, v_{K}\right]
$$

Taking their difference into account, they are expected to be complementary and thus their combination is expected to have a better representation ability. We will empirically prove its effectiveness in Section 6.4.

\subsection{Feature weighting}

Original FV-CNN treat each convolutional descriptor equally and pool them by Fisher Vectors or by VLAD directly. However, in fine-grained image recognition task, the main objects are often confused or even occluded by background and thus descriptors extracted from these useless or even harmful region are unfavorable for recognition. We propose to alleviate this problem by spatially weighting convolutional descriptors with a clear purpose that highlight useful features and suppress useless or harmful ones.

As discussed in Section 3, numerous feature maps are generated spontaneously in each layer in a CNN's pipeline. After a $\mathrm{CNN}$ model has been trained, the activation of the last convolutional layer is a $H \times W \times D$ tensor which can be seen as $H \times W D$-dimensional descriptors when they are used for feature encoding. On the other hand, if we treat it as D feature maps with size $H \times W$, it can be observed that each channel responses to different parts semantically of the input image, as shown in Fig. 3. We pick some feature maps in the last convolutional layer after non-linearity activation and visualize them by simply normalizing their values. Obviously, some feature maps response highly to whole objects, e.g., 15th channel of bird 1, 289th channel of car 2, and some only respond to parts of corresponding objects, e.g., 136th channel of bird 2, 253th channel of dog 2, 109th channel of air 1 while some of them response to noisy background, e.g., 413th channel of bird 2, 192th channel of air 1 , 383th channel of air 2 .

Considering discriminative regions usually have higher activation, and more feature maps will respond to them, we propose to accumulate all feature maps along $D$ channels, then we obtain an activation map with a size of $H \times W$, then this activation map will be applied to each feature map to complete the spatial weighting. Taking VGG-16 as an example, firstly, we extract activation tensor $A \in \mathbb{R}^{H \times W \times D}$ of the last convolutional layer with relu activation, following by a max-pooling of stride 2, we get an intermediate map matrix $W_{\text {in }}$ which usually has size around $\frac{H}{2} \times \frac{W}{2}$. After that, it will be divided by the max value such that all values in it lie in the range $[0,1]$. Then we do a square-rooting operation on the map and obtain the $W_{\text {in }}$ (after dividing by max value), and finally it is resized to $H \times W$ by nearest-neighbor interpolation, and we get the final weighting map $W \in R^{H \times W}$. The whole process is illustrated in (4):

$$
\begin{gathered}
W_{\text {in }}(i, j)=\sum_{t=1}^{D} A(i, j, t), \\
W_{\text {in }}=\sqrt{W_{\text {in }} / \max \left(W_{\text {in }}(\mathbb{S})\right)}, \\
W=\mathbb{N} \mathbb{R}\left(W_{\text {in }}\right) .
\end{gathered}
$$

where $\mathbb{S}$ denotes set of spatial locations of intermediate map and $\mathbb{N N R}$ denotes nearest neighbor interpolationbased resizing.

It deserves to note that this feature weighting method is not limited to the original convolutional activation tensor. In fact, we utilize it to each filtered tensor by multi-pooling as we described in Section 3, and we pool 


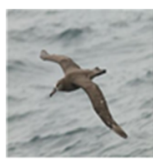

Bird 1

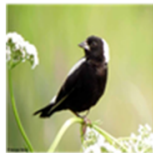

Bird 2

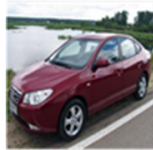

Car 1

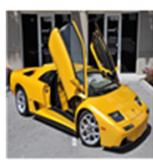

Car 2

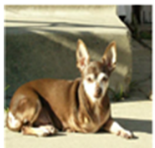

Dog 1

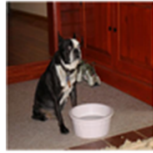

$\operatorname{Dog} 2$

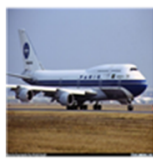

Air 1

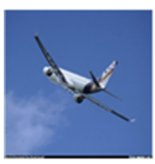

Air 2
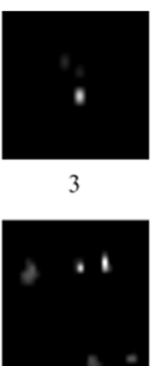

72

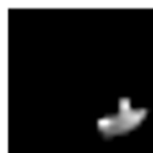

21

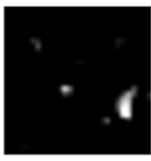

125

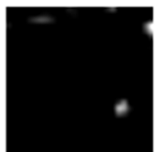

2

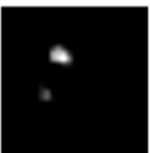

9

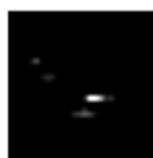

10

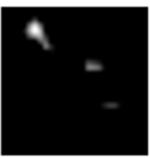

5

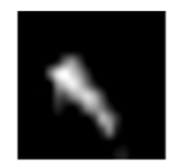

15

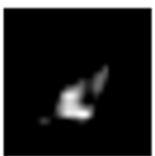

136

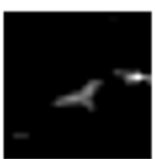

47

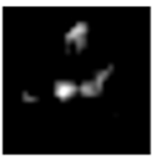

227

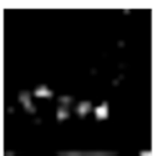

74

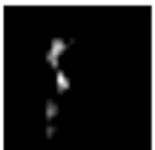

40

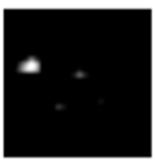

109

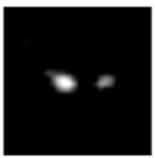

33

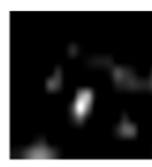

77

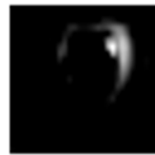

230

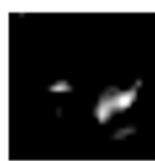

147

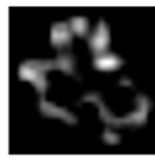

289

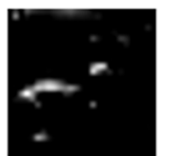

107

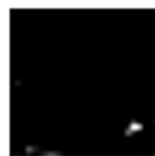

160

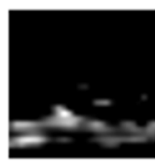

192

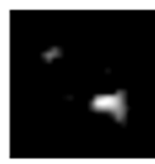

247

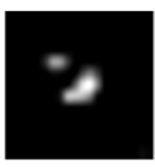

393

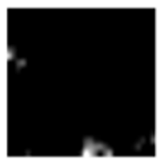

413

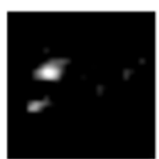

327

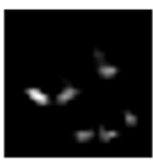

444

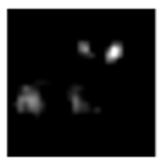

224

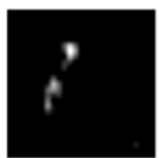

253

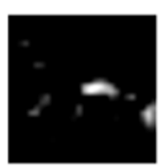

380

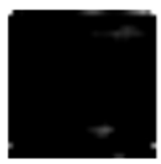

383

Fig. 3 Examples of several images sampled from four datasets [26-28] evaluated in this paper and picked corresponding visualized feature maps the digits below which are the index of channels

all of them together with that weighted tensor from the original activation tensor by VLAD-FV.

\section{Compact FV-CNN}

We propose to concatenate Fisher Vectors and VLAD to form VLAD-FV, which inevitably increases dimension of image representation. Thus, effective dimension reduction deserves to be researched. In view of this problem, we investigate two dimension reduction technologies.

\subsection{MI dimension selection}

A conclusion that strong linear correlation between two dimensions almost does not exist in Fisher Vectors or VLAD is obtained in [10]. Therefore, instead of compressing whole feature dimensions altogether, reducing dimension of Fisher Vectors and VLAD by selecting useful dimensions deserves to be investigated.

We denote image labels as $y$, the Fisher Vectors or VLAD values in the $i$ th dimension as $x_{: i}$, and the 
mutual information as $I(x: i y)$. The MI value represents importance score for each dimension and is computed as:

$$
I\left(x_{: i}, y\right)=H\left(x_{: i}\right)-H\left(x_{: i}, y\right),
$$

Where $H(x)$ is the entropy of a random variable. After the MI values for all dimensions are calculated, dimensions are re-ranked according to their MI values, and if we want to reduce a $D$-dimensional feature to $d$-dimensional, simply select the top $d$ dimensions in sorted list. For more details of this off-the-shelf technology, one may refer to [10].

\subsection{Tensor Sketch approximation}

VLAD and Fisher Vectors are usually reshaped to single vectors for classification. For convenience of lowdimensional analysis, it is desirable to keep the original distribution of each entry as shown in Fig. 4. Inspired by $\mathrm{B}-\mathrm{CNN}, \mathrm{FV}-\mathrm{CNN}$ can be transformed to another representation by matrix multiplication of a FV-CNN and its transposition:

$$
\begin{aligned}
& \Phi_{\mathrm{fv}}(I)=\left[u_{1}, \ldots \ldots ., u_{k}, \sigma_{1}, \ldots \ldots ., \sigma_{k}\right], \\
& \Phi_{v}(I)=\left[v_{1}, \ldots \ldots ., v_{k}\right], \\
& \Phi_{v_{\mathrm{f}} \mathrm{V}}(I)=\left[\Phi_{v}(I), \Phi_{\mathrm{fv}}(I)\right], \\
& \Phi_{v_{\mathrm{f}} \mathrm{v}_{\text {transformed }}}(I)=\Phi_{v_{\mathrm{f}} \mathrm{V}}(I) \times \Phi_{v_{\mathrm{f}} \mathrm{V}}(I)^{T},
\end{aligned}
$$

where $\Phi_{\mathrm{fv}}(I), \Phi_{\nu}(I), \Phi_{v_{-} \mathrm{fv}}(I)$ and $\Phi_{v_{-} \mathrm{fv} \_ \text {transformed }}(I)$ denotes Fisher Vectors, VLAD, VLAD-FV and transformed VLAD-FV, respectively.

The transformed FV-CNN can be viewed as linear kernel machines. Let $X$ and $Y$ denote two sets of Fisher Vectors or VLAD, compare them in image classification using linear classifier such as SVM is operated as follows:

$$
\begin{aligned}
\langle(\Phi(X)),(\Phi(Y))\rangle & =\left\langle\sum_{i \in S_{1}} x_{i} x_{i}^{T}, \sum_{j \in S_{2}} y_{j} y_{j}^{T}\right\rangle \\
& =\sum_{i \in S_{1}} \sum_{j \in S_{2}}\left\langle x_{i}, y_{j}\right\rangle^{2},
\end{aligned}
$$

where $S_{1}$ and $S_{2}$ denote locations of two sets of FV-CNN. It is clear that comparison of entries of FV-CNN of two images is actually a second-order polynomial kernel, thus, methods for low-dimensional approximation of secondorder polynomial kernel can be applied on it. Tensor Sketch is an algorithm, and details of this can be found in [9].

\section{Experiments}

\subsection{Dataset and measurement}

In this section, we will use four fine-grained benchmarks to perform experimental evaluation:

- Caltech-UCSD 2011 bird dataset (cub): it contains 11,788 images of 200 bird species.

- FGVC-aircraft dataset [26] (air): it consists of 10,000 images of 100 aircraft categories.

- FGVC-car dataset [27] (cars): it is composed of 16,185 images of cars from 196 classes.

- Stanford dogs dataset [28] (dogs): 20,580 images with 120 dog species are included in it.

For all datasets, we follow the fixed training and testing split provided by themselves. We do not resort to any object bounding-box and part annotation on both training and testing time, only image labels are used.

Measurement for all experiments is the fraction of correctly predicted images.

\subsection{Networks}

For fair comparison with another method, we use VGG16 [25] to perform experiments on cub, air, and cars while AlexNet [29] for dogs. Both VGG-16 and AlexNet are fine-tuned by B-CNN based on pre-trained models on ImageNet. All of them converge (validation error rate stop decreasing) in less than 60 epoches, and we do not use any data augmentation in fine-tuning.

\subsection{Implementation details}

Images of cub have moderate size, and thus, we directly use the original size of them, while the size of the images from the other three datasets are rather variable and hence we normalize all their images to $448 \times 448 \times 3$ firstly. We double the training data by horizontal flipping, and we average the predictions of a test image and its flipped copy, and we output the class with highest

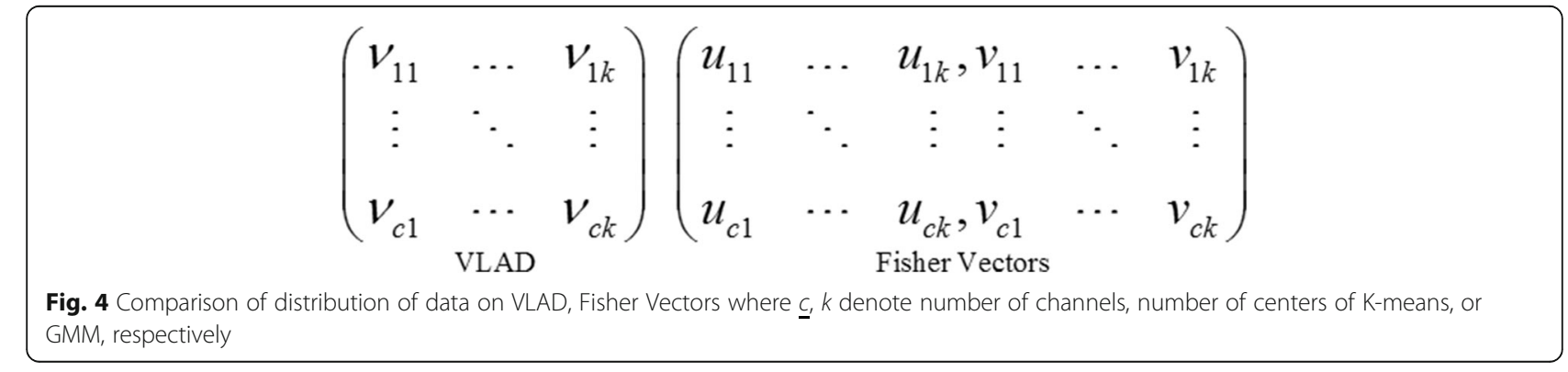


Table 1 Comparison of performance of Fisher Vectors, VLAD, and VLAD-FV

\begin{tabular}{lllll}
\hline Encoding methods & Cub (\%) & Air (\%) & Cars (\%) & Dogs (\%) \\
\hline Fisher Vectors & 79.0 & 82.3 & 83.7 & 64.1 \\
VLAD & 80.8 & 83.0 & 87.1 & 65.8 \\
VLAD-FV & 82.0 & 84.1 & 88.5 & 67.5 \\
\hline
\end{tabular}

score. Once feature extraction for all images is done, one-versus-all linear SVM classifiers will be trained with constant learning hyperparameter $C=1$ to perform recognition. The trained classifiers are recalibrated by changing the SVM bias and rescaling the SVM weight vector such that median scores of the negative and positive training examples are at -1 and +1 , respectively. Number of clusters for GMM and K-means is fixed to 64 .

We implemented all experiments using MatConvNet [30] and VLfeat [31].

\subsection{Results and comparisons 6.4.1 Feature encoding}

First of all, we evaluate the effectiveness of VLAD-FV by comparing the classification accuracies of all datasets using Fisher Vectors, VLAD, and VLAD-FV.

As results shown in Table 1, VLAD-FV consistently outperforms both Fisher Vectors and VLAD alone, hence, in the rest of all experiments, we will use VLADFV as the feature encoding method. Results using VLAD-FV in this part will be used as the baseline in the next experiments.

\subsubsection{Multi-scale feature}

We evaluate the effectiveness of multi-pooling by three comparative experiments: using classical multi-scale strategy by image pyramid, using multi-pooling on single scale image and both of them, i.e., the multi-pooling of image pyramids. Experimental results are shown in Table 2 from which we observe using multi-pooling on single image and on image pyramids that can boost recognition ability. For image pyramids, we use six scales with re-scaling factor $[0.5,0.75,1,1.25,1.5,1.75]$. For cub, the factor 1 represents original image size, and for the other three datasets, it represents images rescaled to $448 \times 448 \times 3$ beforehand. We use six levels of multi-pooling because we find

Table 2 Evaluation of multi-pooling

\begin{tabular}{lllll}
\hline Multi-scale strategy & Cub (\%) & Air (\%) & Cars (\%) & Dogs (\%) \\
\hline Baseline & 82.0 & 84.1 & 88.5 & 67.5 \\
Image pyramid & 84.9 & 85.8 & 90.3 & 69.7 \\
Multi-pooling of single image & 84.2 & 85.2 & 89.9 & 68.6 \\
Multi-pooling of image pyramid & 85.6 & 86.5 & 91.3 & 71.4 \\
\hline
\end{tabular}

that with this parameter, we achieve best performance in all datasets.

\subsubsection{Descriptors weighting}

Two experiments are presented in this part. Firstly, we evaluate descriptor weighting alone and then we evaluate using descriptors weighting along with multi-pooling strategy. In Table 3, we see the effectiveness of descriptors weighting, and its concordance with multi-pooling.

\subsubsection{Dimension reduction}

Experiments in this part is composed of four groups: dimension reduction by $\mathrm{MI}$, dimension reduction by Tensor Sketch, dimension reduction by jointly using MI and Tensor Sketch, and widely used principal component analysis (PCA) where we project original descriptors from 512-dimensional to 24-dimensional such that the dimension of the final feature vector will be $24 \times 64+24 \times 2 \times 64=4608$ and being comparable to other methods. All operations here are based on the best practices used above, i.e., multi-pooling of image pyramid with spatially weighted descriptors encoded by VLAD-FV. For MI selection, we present two results using two different numbers of selected dimensions because we find that when we carefully select useful dimensions $(69,992$ for VGG-16 and 40,000 for AlexNet), the accuracy may even improve while if we simply select a few dimension (4096 here) the performance drops violently.

From Table 4, we can see that jointly using tensor sketch and MI consistently outperforms solely using either of them. It also has a better performance than widely used dimension reduction method PCA.

\subsubsection{Comparison with other methods}

Comparison with other methods in this part is twofold; in terms of accuracy and jointly considering accuracy and dimension of feature vector. For later, we define an index-termed discriminative per dimension and abbreviated to DPD, which can be explained as discriminative power of a single dimension for better evaluating the effectiveness of dimension reduction method. This is calculated as follows:

Table 3 Evaluation of descriptors weighting

\begin{tabular}{lllll}
\hline Methods & $\begin{array}{l}\text { Cub } \\
(\%)\end{array}$ & $\begin{array}{l}\text { Air } \\
(\%)\end{array}$ & $\begin{array}{l}\text { Cars } \\
(\%)\end{array}$ & $\begin{array}{l}\text { Dogs } \\
(\%)\end{array}$ \\
\hline Baseline & 82.0 & 84.1 & 88.5 & 67.5 \\
Descriptors weighting & 83.6 & 85.4 & 90.3 & 68.7 \\
$\begin{array}{l}\text { Descriptors weighting+multi- } \\
\text { pooling }\end{array}$ & 86.3 & 87.5 & 92.1 & 72.3 \\
\hline
\end{tabular}


Table 4 Evaluation of dimension reduction. For dogs, we use AlexNet and thus the dimension of full vector of this is 49,152

\begin{tabular}{llllll}
\hline Strategy & Cub (\%) & Air (\%) & Cars (\%) & Dogs (\%) & Dimension \\
\hline Full vector & 86.3 & 87.5 & 92.1 & 72.3 & $98,304 / 49,152$ \\
Tensor Sketch & 84.9 & 83.4 & 88.9 & 69.6 & 12,288 \\
MI Selection 1 & 75.3 & 73.6 & 81.8 & 63.9 & 4,906 \\
MI Selection 2 & 86.4 & 87.7 & 92.4 & 72.6 & $69,992 / 40,000$ \\
Tensor Sketch + Ml & 84.5 & 82.5 & 87.5 & 68.4 & 4,906 \\
PCA & 82.3 & 81.0 & 85.2 & 65.6 & 4,608 \\
\hline
\end{tabular}

$$
\mathrm{DPD}=C \times \frac{\mathrm{ACC}}{D}
$$

where $A C C$ and $D$ denote classification accuracy and dimension of feature vector of corresponding method, respectively. $C$ is a constant which we set to 8000 in this paper.

\subsection{Discussion}

Based on the above experimental results, several conclusions can be obtained:

- VLAD-FV can be used instead as an off-the-shelf encoding method to improve classification accuracy when Fisher Vector or VLAD is going to be used. Difference of formulation and used visual dictionaries make Fisher Vector and VLAD being complementary and thus can be concatenated to form a more powerful representation.

- The proposed multi-pooling of convolutional activation tensor can either be used alone which is very efficient or with traditional image pyramid multiscale strategy which has better performance in terms of classification accuracy.
- Descriptor weighting can boost performance because it highlights discriminative feature and suppresses useless background information. For better illustrating, the reason why this simple weighting is effective, we sample a few images from all evaluated datasets and visualize their activation map referenced in (4) and activation map after weighting which is shown in Fig. 5. We can see that compared with original activation maps, the weighted maps respond more on discriminative parts, and activation on background are attenuated.

- From Table 5, we conclude that while tensor sketch sorts to conserve discriminative power using as few dimension as possible, MI emphasizes on selecting the most useful information in a given dimension. Thus, jointly using them is preferred when both discriminative power and compact representation are pursued. Although both MI dimension selection and tensor sketch approximation are existing methods, there are two points that deserve to be noted: firstly, to the best of our knowledge, we are the first to apply tensor sketch on Fisher Vectors and VLAD (for VLAD-FV, tensor sketch is applied on VLAD part and Fisher Vectors part separately,

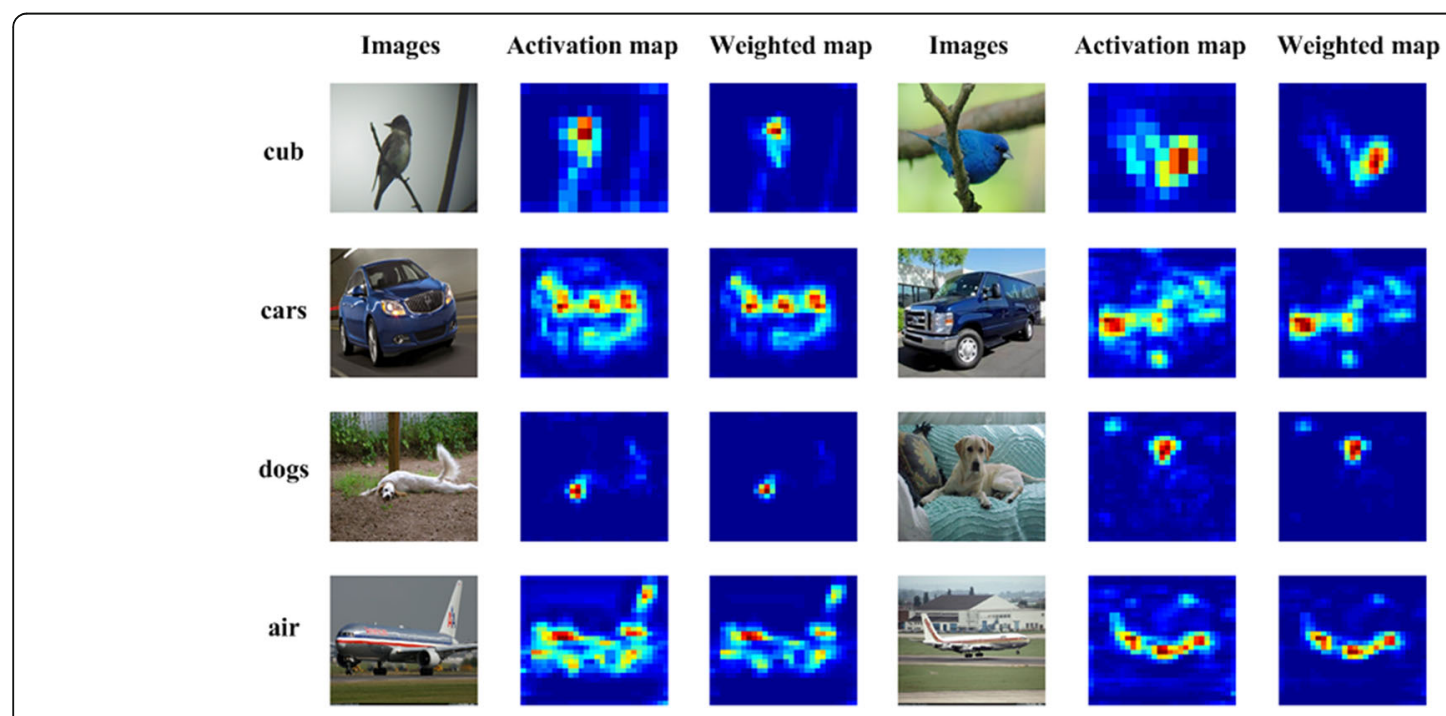

Fig. 5 Examples sampled from four datasets and their corresponding visualized activation map and weighted map 
Table 5 Comparison of performance of our methods with some recent state-of-the-art methods in cub. BBox, parts denote bounding-box and parts annotation respectively

\begin{tabular}{|c|c|c|c|c|c|c|}
\hline Methods & Train phase & Test phase & Dim. & Model & Acc. & DPD \\
\hline & & Dataset: cub & & & & \\
\hline Part-stacked CNN [1] & BBox+Parts & BBox & 4,096 & Part-Stacked CNN & $76.2 \%$ & 1.484 \\
\hline Deep LAC [2] & BBox+Parts & BBox & 12,288 & Alex-Net & $80.3 \%$ & 0.521 \\
\hline PN-CNN [3] & BBox+Parts & $\mathrm{n} / \mathrm{a}$ & 13,512 & Alex-Net & $85.4 \%$ & 0.506 \\
\hline PG-alignment [4] & BBox & $n / a$ & 126,976 & VGG-19 & $82.8 \%$ & 0.052 \\
\hline Symbolic [5] & BBox & BBox & 20,992 & Shallow feature: SIFT & $59.4 \%$ & 0.226 \\
\hline Cross layer pooling[6] & BBox & BBox & & Alex-Net & $73.5 \%$ & 1.436 \\
\hline Mask-CNN [12] & Parts & $\mathrm{n} / \mathrm{a}$ & 8192 & VGG-16+FCN & $85.4 \%$ & 0.834 \\
\hline Spatial transformer CNN [32] & $\mathrm{n} / \mathrm{a}$ & $n / a$ & & ST-CNN & $84.1 \%$ & 1.643 \\
\hline Bilinear CNN [8] & $\mathrm{n} / \mathrm{a}$ & $n / a$ & 262,144 & VGG-16+VGG-M & $84.1 \%$ & 0.026 \\
\hline Compact bilinear CNN [24] & $\mathrm{n} / \mathrm{a}$ & $\mathrm{n} / \mathrm{a}$ & 8,192 & VGG-16 & $84.0 \%$ & 0.820 \\
\hline PD+SWFV [14] & $\mathrm{n} / \mathrm{a}$ & $n / a$ & 69,632 & VGG-16 & $84.5 \%$ & 0.097 \\
\hline SCDA [13] & $\mathrm{n} / \mathrm{a}$ & $\mathrm{n} / \mathrm{a}$ & & VGG-16 & $80.5 \%$ & 1.572 \\
\hline Ours & $\mathrm{n} / \mathrm{a}$ & $\mathrm{n} / \mathrm{a}$ & 69,992 & VGG-16 & $86.4 \%$ & 0.099 \\
\hline \multirow[t]{2}{*}{ Ours (compact vector) } & $\mathrm{n} / \mathrm{a}$ & $\mathrm{n} / \mathrm{a}$ & & VGG-16 & $84.5 \%$ & 1.650 \\
\hline & & Dataset: air & & & & \\
\hline Symbolic [5] & BBox & BBox & 20,992 & Shallow feature: SIFT & $72.5 \%$ & 0.276 \\
\hline Re-Fisher Vector [34] & $\mathrm{n} / \mathrm{a}$ & $n / a$ & 655,360 & Shallow feature: SIFT & $81.5 \%$ & 0.001 \\
\hline Bilinear CNN [8] & $\mathrm{n} / \mathrm{a}$ & $\mathrm{n} / \mathrm{a}$ & 262,144 & VGG-16+VGG-M & $83.9 \%$ & 0.0256 \\
\hline Ours (Full Vector + MI 2) & $\mathrm{n} / \mathrm{a}$ & $\mathrm{n} / \mathrm{a}$ & 69,992 & VGG-16 & $87.7 \%$ & 0.100 \\
\hline \multirow[t]{2}{*}{ Ours (compact vector) } & $\mathrm{n} / \mathrm{a}$ & $\mathrm{n} / \mathrm{a}$ & & VGG-16 & $82.5 \%$ & 1.611 \\
\hline & & Dataset: cars & & & & \\
\hline Symbolic [5] & BBox & BBox & 20,992 & Shallow feature: SIFT & $78.0 \%$ & 0.297 \\
\hline PG-Alignment [4] & BBox & $\mathrm{n} / \mathrm{a}$ & 126,976 & VGG-19 & $92.6 \%$ & 0.058 \\
\hline Re-Fisher Vector [34] & $\mathrm{n} / \mathrm{a}$ & $\mathrm{n} / \mathrm{a}$ & 655,360 & Shallow feature: SIFT & $82.7 \%$ & 0.011 \\
\hline Bilinear CNN [8] & $\mathrm{n} / \mathrm{a}$ & $\mathrm{n} / \mathrm{a}$ & 262,144 & VGG-16+VGG-M & $91.3 \%$ & 0.028 \\
\hline Ours & $\mathrm{n} / \mathrm{a}$ & $\mathrm{n} / \mathrm{a}$ & 69,992 & VGG-16 & $92.4 \%$ & 0.106 \\
\hline \multirow[t]{2}{*}{ Ours (compact vector) } & $n / a$ & $\mathrm{n} / \mathrm{a}$ & & VGG-16 & $87.5 \%$ & 1.709 \\
\hline & & Dataset: dogs & & & & \\
\hline Symbolic [5] & BBox & BBox & 20,992 & Shallow feature: SIFT & $45.6 \%$ & 0.174 \\
\hline Selective pooling [35] & BBox & BBox & 163,840 & Shallow feature: SIFT & $52.0 \%$ & 0.025 \\
\hline Re-Fisher Vector [34] & $\mathrm{n} / \mathrm{a}$ & $\mathrm{n} / \mathrm{a}$ & 327,680 & Shallow feature: SIFT & $52.9 \%$ & 0.013 \\
\hline NAC[33] & $\mathrm{n} / \mathrm{a}$ & $\mathrm{n} / \mathrm{a}$ & & Alex-Net & $68.6 \%$ & 1.340 \\
\hline PD+SWFV [14] & $\mathrm{n} / \mathrm{a}$ & $\mathrm{n} / \mathrm{a}$ & 36,864 & Alex-Net & $71.9 \%$ & 0.156 \\
\hline Ours & $\mathrm{n} / \mathrm{a}$ & $\mathrm{n} / \mathrm{a}$ & 40,000 & Alex-Net & $72.6 \%$ & 0.145 \\
\hline Ours (compact vector) & $n / a$ & $\mathrm{n} / \mathrm{a}$ & & Alex-Net & $68.4 \%$ & 1.335 \\
\hline
\end{tabular}

The ' $n / a$ ' entries in the table means that the results are not available

and their approximated vectors will be concatenated); secondly, we are the first to jointly apply these two technologies to compact final image representation.

- Some state-of-the-art methods are still very competitive, but our framework has its own advantages. Compared with $[3,4,12]$, our framework do not rely on any bounding-box or part annotations and achieve similar or even better results; compared with [8, 24, 32], because we use FV-CNN as our baseline, our framework has better ability to incorporate multi-scale information and achieve better performance in the end; compared with [14, 33], we do not need to train an extra part detector, which are always non-trivial. 


\section{Conclusions}

In conclusion, the main contribution of this paper lie in four aspects: firstly, we propose a novel multi-scale strategy which can be utilized efficiently alone or together with classical image pyramids strategy which has better performance in terms of classification accuracy; secondly, we propose VLADFV to encode deep convolutional descriptors by concatenating Fisher Vectors and VLAD, resulting in better performance than only using either of them; thirdly, VLAD-FV is rather high-dimensional and thus we apply two dimension reduction methods to compact final image representation; last, but not the least, we propose a feature weighting method in descriptor level, which further enhances the performance.

\section{Acknowledgements}

None.

\section{Funding}

None.

\section{Authors' contributions}

WZ constructed the main ideas of the research, carried out most experiments, and drafted the original manuscript. JY and WS took part in the examination of the study and participated in revising the manuscript. TF and DD offered useful suggestions in conducting experiments and drafting the manuscript. All authors read and approved the final manuscript.

\section{Competing interests}

The authors declare that they have no competing interests.

\section{Publisher's Note}

Springer Nature remains neutral with regard to jurisdictional claims in published maps and institutional affiliations.

\section{Author details}

${ }^{1}$ School of Electronic Information, Wuhan University, Wuhan, China. ${ }^{2}$ School of Remote Sensing and Information Engineering, Wuhan University, Wuhan, China.

Received: 14 September 2016 Accepted: 14 March 2017

Published online: 08 April 2017

\section{References}

1. S Huang, Z Xu, D Tao, Y Zhang, Part-stacked CNN for fine-grained visual categorization, in Proceedings of the IEEE Conference on Computer Vision and Pattern Recognition (CVPR), 2016

2. D Lin, X Shen, C Lu, J Jia, Deep LAC: Deep localization, alignment and classification for fine-grained recognition, in Proceedings of the IEEE Conference on Computer Vision and Pattern Recognition(CVPR), 2015, pp. $1666-1674$

3. S Branson, G Van Horn, S Belongie, P Perona, Bird specifies categorization using pose normalized deep convolutional nets, in Proceedings of The British Machine Vision Conference (BMVC), 2014

4. J Krause, H Jin, J Yang, L Fei Fei, Fine-grained recognition without part annotations, in Proceedings of the IEEE Conference on Computer Vision and Pattern Recognition(CVPR), 2015, pp. 5546-5555

5. Y Chai, V Lempitsky, A Zisserman, Symbiotic segmentation and part localization for fine-grained categorization. IEEE Int Conf Comp Vision 163(3), 321-328 (2013)

6. L Liu, C Shen, A van den Henge, The treasure beneath convolutional layers: Crossconvolutional-layer pooling for image classification, in Proceedings of the IEEE Conference on Computer Vision and Pattern Recognition(CVPR), 2014, pp. 4749-4757

7. M Cimpo, S Maji, A Vedaldi, Deep filter banks for texture recognition, description and segmentation. Int J Comput Vis 188(1), 65-94 (2016)

8. T.Y. Lin, A. RoyChowdhury, S. Maji. Bilinear CNN models for fine-grained visual recognition. In IEEE International Conference on Computer Vision (ICCV), 1449-1457, 2015
9. N Pham, R Pagh, Fast and scalable polynomial kernels via explicit feature maps, in Proceedings of the 19th ACM SIGKDD international conference on Knowledge discovery and data mining, 2013, pp. 239-247

10. Y Zhang, JX Wu, JF Cai, Compact representation of high-dimensional feature vectors for large-scale image recognition and retrieval. IEEE Trans Image Process 25(5), 2407-2419 (2016)

11. A Borji, MM Cheng, H Jiang, J Li, Salient object detection: a benchmark. IEEE Trans Image Process 24(12), 5706-5722 (2015)

12. X. S. Wei, C-W. Xie, J. X. Wu. Mask-CNN: Localizing parts and selecting descriptors for fine-grained image recognition. arxiv.org, 1605.06878, 2016.

13. X.S. Wei, J.H Luo and J.X. Wu. Selective convolutional descriptor aggregation for fine-grained image retrieval. arXiv:1604.04994,2016.

14. X Zhang, H Xiong, W Zhou, W Lin, Q Tian, Picking deep filter responses for fine-grained image recognition, in Proceedings of the IEEE Conference on Computer Vision and Pattern Recognition(CVPR), 2016

15. F. Perronnin, J. Sánchez, and T. Mensink. Improving the fisher kernel for large-scale image classification. In Europeon Conference on Computer Vision(ECCV), 143-156. Springer, 2010.

16. F Perronnin, C Dance, Fisher kernels on visual vocabularies for image categorization, in Proceedings of the IEEE Conference on Computer Vision and Pattern Recognition(CVPR), 2007, pp. 1-8

17. H Jégou, M Douze, C Schmid, P Pérez, IEEE Conference on Computer Vision and Pattern Recognition(CVPR), 2010, pp. 3304-3311

18. R Arandjelovi'c, A Zisserman, All about VLAD, in Proceedings of the IEEE Conference on Computer Vision and Pattern Recognition(CVPR), 2013

19. A. Razavian, H. Azizpour, J. Sullivan, and S. Carlsson. CNN Features off-the-shelf: an astounding baseline for recognition. CoRR, vol. abs/1403.6382, 2014.

20. K Chatfield, K Simonyan, A Vedaldi, A Zisserman, Return of the devil in the details: delving deep into convolutional nets, in Proceedings of The British Machine Vision Conference (BMVC), 2014

21. D. G. Lowe. Object recognition from local scale-invariant features. In The proceedings of the seventh IEEE international conference on Computer Vision (ICCV). 2:1150-1157,1999

22. N Dalal, B Triggs, Histograms of oriented gradients for human detection Proc IEEE Conf Comput Vis Pattern Recognit 1, 886-893 (2005)

23. T Ojala, M Pietikinen, D Harwood, A comparative study of texture measures with classification based on feature distributions. Pattern Recogn 29(1), 51-59 (1998)

24. Y Gao, O Beijbom, N Zhang, T Darrell, Compact bilinear pooling, in Proceedings of the IEEE Conference on Computer Vision and Pattern Recognition (CVPR), 2016

25. K. Simonyan and A. Zisserman. Very deep convolutional networks for largescale image recognition. CoRR abs/1409.1556,2014.

26. S. Maji, E. Rahtu, J. Kannala, M. Blaschoko and A. Vedaldi. Fine-grained visual classification of aircraft. arXiv:1306.5151,2013

27. J Krause, M Stark, J Deng, L Fei-Fei, 3d object representation for fine-grained categorization, in 3D Representations and Recognition Workshop, The proceedings of the seventh IEEE international conference on Computer Vision (ICCV), 2013

28. A Khosla, N Jayadevaprakash, B Yao, L Fei-Fei, Novel dataset for fine-grained image categorization, in Proceedings of the IEEE Conference on Computer Vision and Pattern Recognition (CVPR), 2011

29. A. Krizhevsky, I. Sutskever and G. Hinton. ImageNet classification with deep convolutional neural networks. Advances in Neural Information Processing Systems, 25(2):1097-1105,2012

30. A. Vedaldi and K. Lenc. MatConvNet: Convolutional neural networks for MATLAB, 2014. Software available at http://www.vlfeat.org/matconvnet/.

31. A. Vedaldi and B. Fulkerson. VLFeat: An open and portable library of computer vision algorithms, 2008. Software available at http://www.vlfeat.org/.

32. M. Jaderberg, K. Simonyan, A. Zisserman and K. Kavukcuoglu. Spatial transformer networks. In Conference and Workshop on Neural Information Processing Systems (NIPS). 2008-2016, 2015

33. M Simon, E Rodner, Neural activation constellations: unsupervised part model discovery with convolutional networks, in The proceedings of the seventh IEEE international conference on Computer Vision (ICCV), 2015, pp. 1143-1151

34. P. H. Gosselin, N. Murray, H. Jégou and F. Perronnin. Revisiting the fisher vector for fine-grained classification. Pattern Recognition Letters, Elsevier, 49, pp.92-98, 2014

35. G Chen, J Yang, H Jin, E Shechtman, J Brandt, Selective pooling vector for fine-grained recognition, in IEEE Winter Conference on Application of Computer Vision (WCAC), 2015, pp. 860-867 